\title{
CULICIDAE (DIPTERA: CULICOMORPHA) DA AMAZÔNIA OCIDENTAL BRASILEIRA: QUERARI.
}

\author{
Rosa Sá Gomes HUTCHINGS ', Maria Anice Mureb SALLUM ², \\ Ruth Leila Menezes FERREIRA ${ }^{1}$
}

\begin{abstract}
Resumo - Foram coletados adultos e imaturos de Culicidae (Diptera: Culicomorpha) em Querari, Município de São Gabriel da Cachoeira, Estado do Amazonas, Brasil. Os adultos foram capturados utilizando armadilhas (CDC e Malaise) e com puçá (varredura). Os imaturos foram coletados em poças de água (no solo e em pedras) e nas axilas de bromélias epífitas e terrestres. Apresenta-se uma lista das espécies da família Culicidae, incluindo 42 novos registros de distribuição para a região. As espécies encontradas pertencem aos gêneros Ochlerotatus, Anopheles, Coquillettidia, Culex, Haemagogus, Johnbelkinia, Limatus, Psorophora, Sabethes, Trichoprosopon, Uranotaenia e Wyeomyia.
\end{abstract}

Palavras-Chave: Culicidae, Amazônia, Distribuição

\section{Culicidae (Diptera: Culicomorpha) from the Western Brazilian Amazon: Querari.}

Abstract - Adult and immature specimens of Culicidae (Diptera: Culicomorpha) were collected in Querari, Municipality of São Gabriel da Cachoeira, State of Amazonas, Brazil. Adults were captured using traps (CDC and Malaise) and nets (sweeping). Larvae and pupae were collected from puddles of water (on the ground and on rocks) and from leaf axils of arboreal and terrestrial Bromeliales. A species list of the family Culicidae is presented which includes 42 new distributional records for the region. The species found belong to the genera Ochlerotatus, Anopheles, Coquillettidia, Culex, Haemagogus, Johnbelkinia, Limatus, Psorophora, Sabethes, Trichoprosopon, Uranotaenia and Wyeomyia.

Key-Words: Culicidae, Amazonia, Distribution

\section{INTRODUÇÃO}

Existem mais de 3200 espécies de Culicidae descritas em todo o mundo, e esse número cresce aproximadamente $5 \%$ a cada década (Ward, 1992). Os culicídeos têm distribuição mundial com múltiplas espécies presentes na região Neotropical. Dados de distribuição geográfica são essenciais para melhorar os conhecimentos da sistemática de mosquitos, bem como da epidemiologia das doenças veiculadas por esses animais (Nielsen, 1980). Dessa maneira, o conhecimento da biodiversidade de Culicidae é de interesse epidemiológico pois possibilita melhor compreensão da dinâmica da transmissão dos agentes infecciosos e do papel vetor das espécies de Culicidae, facilitando a adoção de medidas de controle. No entanto, sabe-se pouco sobre a fauna e distribuição dos mosquitos na região amazônica. Cerqueira (1961), em trabalho

'Coordenação de Pesquisas em Entomologia, Instituto Nacional de Pesquisas da Amazônia, Caixa Postal 478, Manaus, Amazonas, Brasil 69011-970

${ }^{2}$ Núcleo de Pesquicas Taxonômica e Sistemática em Entomologia Médica, Departamento de Epidemiologia, Faculdade de Saúde Pública, Universidade de São Paulo, Av. Dr. Arnaldo, 715, São Paulo, São Paulo, Brasil 01246-904 
pioneiro, assinalou a presença de 148 espécies que foram coletadas em 24 localidades do estado do Amazonas. Posteriormente, Xavier \& Mattos (1976) acrescentaram 25 novos registros, elevando para 175 o número de espécies conhecidas em 114 localidades.

A região amazônica, por sua complexa estrutura geográfica e difícil acesso, possui algumas áreas cuja fauna de Culicidae é ainda, praticamente, desconhecida. Entre elas, vale citar o extremo norte do estado do Amazonas, divisa entre o Brasil e a Colômbia, mais especificamente, a localidade de Querari que está situada no município de São Gabriel da Cachoeira.

Neste trabalho registramos as espécies de Culicidae coletadas em Querari, durante período de 40 dias, incluindo informações sobre os métodos de coleta e dos criadouros onde foram encontradas as formas imaturas. O objetivo deste trabalho é o de servir como referência para futuros levantamentos da fauna de Culicidae daquela região.

\section{MATERIAL E MÉTODOS}

As coletas foram realizadas na sede do Pelotão de Fronteira do Exército Brasileiro ( $\left.2^{\circ} / \mathrm{CEF} / 5^{\circ} \mathrm{BIS}\right)$ localizado em Querari, Município de São Gabriel da Cachoeira, margem esquerda do Rio Uaupés, Estado do Amazonas, Brasil (0105' N; 69 ${ }^{\circ} 51^{\prime}$ ' W) (Fig. 1). A referida região inclui os rios de águas escuras que formam a bacia hidrográfica do Alto rio Negro, tendo os rios Uaupés e Içana, como principais afluentes. O clima da região, segundo a classificação de Koopen (1948) é do tipo AF, quente e úmido, com temperatura média registrada de $25^{\circ} \mathrm{C}$ e precipitação anual superior a $850 \mathrm{~mm}$. Não existe estação seca na área. Os meses de maior precipitação na área do rio Uaupés são maio e dezembro. A umidade relativa apresenta-se elevada o ano todo, com médias mensais de $85-90 \%$. A área caracteriza-se por seu relevo ondulado, apresentando cobertura vegetal com parcelas dispersas de campinarana de porte arbóreo, densa ou aberta com árvores grandes, altas e dispersas. Geralmente as partes mais elevadas têm solo argiloso e as mais baixas, arenoso. Apresenta sub-bosque denso com numerosas Piperaceae, Melastomataceae, Cyclantaceae, Musaceae e epífitas, além de bromélias terrestres, líquens, pteridófitas e frequentes agrupamentos de palmeiras (Radambrasil, 1976).

Pelo seu posicionamento junto ao extremo Norte da região mais ocidental do Brasil, são muitas as dificuldades de acesso ao local devido à presença de inúmeras cachoeiras, principalmente, no trecho Querari-Iauaretê. A região apresenta baixa densidade demográfica, ocorrendo apenas pequenos núcleos populacionais, que ocupam áreas marginais ao longo dos rios onde predominam aldeias indígenas. $\mathrm{O}$ principal meio de transporte é o fluvial. Podem ser considerados como meios alternativos, de menor expressão, devido ao quantitativo da carga transportada, aviões e helicópteros de instituições do 
Ministério da Defesa do governo brasileiro.

As coletas foram realizadas no período de 7 de abril a 19 de maio de 1993. As capturas dos mosquitos adultos tiveram lugar no interior da mata, ao longo de trilhas já existentes. Utilizaram-se armadilhas com isca luminosa tipo CDC colocadas a um metro de altura do solo, armadilha de interceptação de vôo tipo Malaise, e varredura com puçá. As armadilhas foram distribuídas em áreas de mata, ao lado de igarapés, e as coletas realizadas entre as 18:00 - 6:00hs. As coletas dos estágios imaturos foram feitas, manualmente, com o auxílio de pipetas, peneiras e conchas. As larvas e pupas encontradas em fitotelmata (sensu Frank \& Lounibos, 1983) foram retiradas por sucção da água das axilas foliares. Imaturos também foram coletados em poças de água presentes em cavidades de rochas próximas de cachoeiras e em poças no solo da mata. A temperatura dos criadouros variou de 23 a $25^{\circ} \mathrm{C}$. Parte das larvas coletadas, foram criadas individualmente de modo a possibilitar e verificar a identificação dos estágios adultos, o restante foi fixado em álcool etílico $70 \%$.

As identificações foram realizadas no Laboratório de Taxonomia, Núcleo de Pesquisas Taxonômicas e Sistemática em Entomologia Médica (NUPTEM-FSPUSP) utilizando-se chaves dicotômicas contidas em Lane (1953), Forattini (1965), Zavortink (1979), e Berlin \& Belkin (1980). Sempre que possível

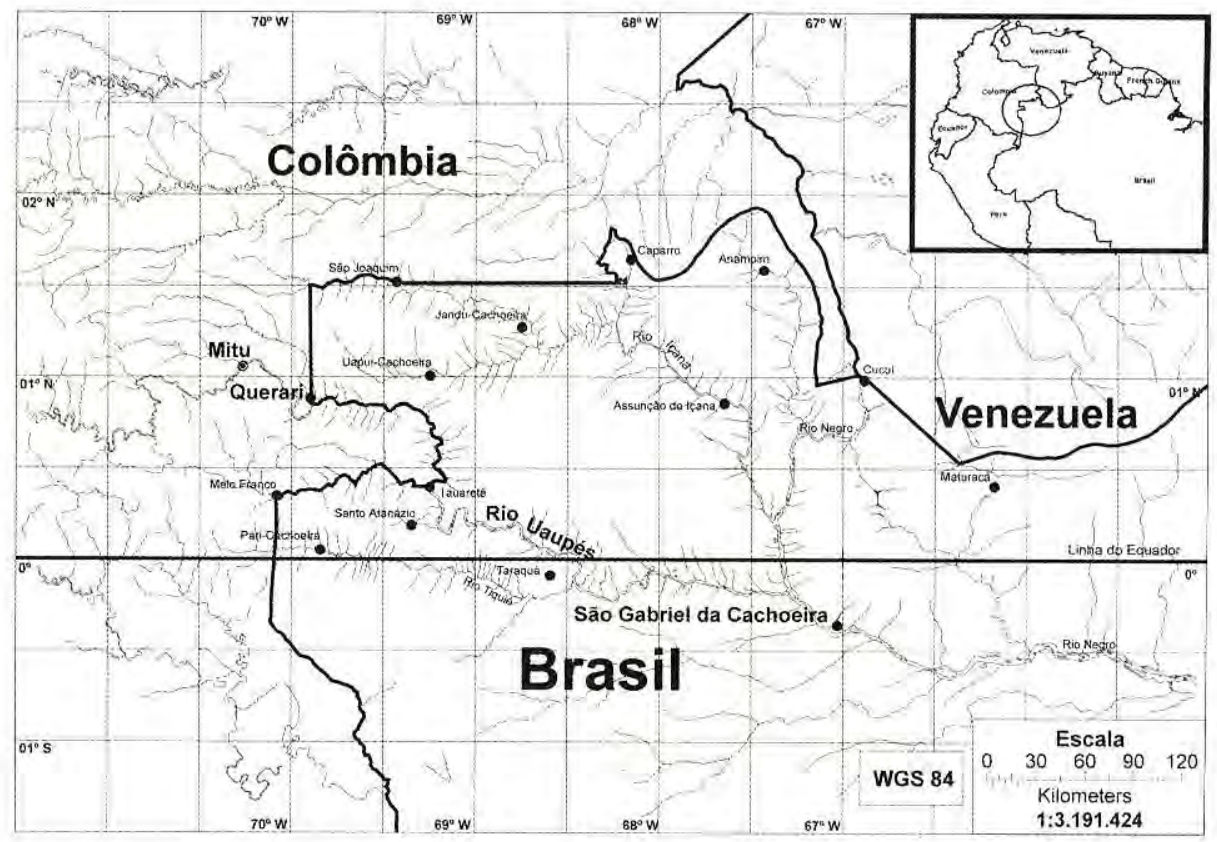

Figura 1. Localização de Querari, Município de São Gabriel da Cachoeira, Estado do Amazonas, Brasil. 
foram examinados caracteres de Culicidae, sendo: 268 correspondentes anatômicos da genitália masculina e a adultos e 102, imaturos, Identificaramdo cibário feminino de modo a obter se 44 espécies e 12 morfotipos, identificação mais precisa dos taxa distribuídos em 12 gêneros (Tab. 1). Vale encontrados. Os exemplares estão assinalar que algumas identificações não depositados na Coleção de puderam ser exatas pela ausência de Invertebrados do Instituto Nacional de indivíduos do sexo masculino, cujas Pesquisas da Amazônia (INPAManaus) e na Coleção Entomológica de Referência da Faculdade de Saúde Pública, Universidade de São Paulo (FSP-USP).

\section{RESULTADOS}

Foram coletados 370 exemplares genitálias, geralmente, possuem caracteres anatômicos que possibilitam o diagnóstico específico. Estes exemplares foram identificados como "morfotipos", procurando-se sempre assinalar a espécie com a qual se assemelha.

Os adultos foram capturados com armadilhas tipo CDC (Anopheles, Culex,

Tabela 1. Relação das espécies de Culicidae coletadas em Querari, Estado do Amazonas, Brasil, distribuídas de acordo com a classificação atual de Culicidae.

\begin{tabular}{|c|c|c|c|}
\hline Tribo & Gênero & Subgênero & Espécie \\
\hline \multirow[t]{2}{*}{........ } & Anopheles & Stethomyia & An. nimbus (Theobald) \\
\hline & & Nyssorhynchus & An. oswaldoi (Peryassu) \\
\hline \multirow[t]{12}{*}{ Aedini } & Ochlerotatus & Howardina & Oc. arborealis (Bonne-Wepster \& Bonne) \\
\hline & & Ochlerotatus & Oc. fluviatilis (Lutz) \\
\hline & & & Oc. hastatus (Dyar) \\
\hline & & & Oc. hastatus / oligopistus \\
\hline & & & Oc. hortator (Dyar \& Knab) \\
\hline & & & Oc. serratus (Theobald) \\
\hline & & & Oc. serratus / nubilus \\
\hline & Haemagogus & Conopostegus & Hg. leucocelaenus (prox.) \\
\hline & Psorophora & Janthinosoma & Ps, albigenu (Peryassú) \\
\hline & & & Ps. albipes (Theobald) \\
\hline & & & Ps. amazonica Cerqueira \\
\hline & & & Ps. ferox (Von Humboldt) \\
\hline \multirow[t]{3}{*}{ Culicini } & Culex & Aedinus & Cx. guyanensis / clastrieri \\
\hline & & Melanoconion & Cx. alogistus Dyar \\
\hline & & & Cx. coppenamensis (prox.) \\
\hline
\end{tabular}


Tabela 1. Continuação

\begin{tabular}{|c|c|c|c|}
\hline Tribo & Gênero & Subgênero & Espécie \\
\hline & & & Cx. crybda Dyar \\
\hline & & & Cx. distinguendus Dyar \\
\hline & & & Cx, educator (prox.) \\
\hline & & & Cx. vaxus Dyar \\
\hline & & & Cx. vaxus (morfotipo 1) \\
\hline & & & Cx. vaxus (morfotipo 2) \\
\hline & & & Cx. lacertosus Komp \& Rozeboom \\
\hline & & & Cx. pedroi Sirivanakarn \& Belkin \\
\hline & & & Cx, theobaldi (Lutz) \\
\hline & & & Cx. vomerifer Komp \\
\hline & & & Cx. zeteki Dyar \\
\hline & & & Cx. evansae (prox.) \\
\hline & & & Cx. (Mel.) spp. \\
\hline & & Culex & Cx. declarator Dyar \& Knab \\
\hline & & & Cx. mollis Dyar \& Knab \\
\hline & & & Cx. (Cux.) spp grupo Coronator \\
\hline & & & CX. (Cux.) spp. \\
\hline & & & Cx. surinamensis Dyar \\
\hline & & Carrollia & Cx. urichii (Coquillett) \\
\hline & & & Cx. anduzei / urichil \\
\hline \multirow{20}{*}{$\begin{array}{l}\text { Mansoniini } \\
\text { Sabethini }\end{array}$} & Coquillettidia & Rhynchotaenia & Cq. arribalzagae (Theobald) \\
\hline & Johnbelkinia & & Jh. longipes (Fabricius) \\
\hline & Limatus & & Li. durhamii (Theobald) \\
\hline & & & Li. flavisetosus De Oliveira Castro \\
\hline & & & Li. pseudomethysticus (Bonne-Wepster \& Bonne \\
\hline & Sabethes & Sabethes & Sa. atbiprivus (Theobald) \\
\hline & & & Sa. cyaneus (Fabricius) \\
\hline & & & Sa. purpureus (Theobald) \\
\hline & & Sabethoides & Sa. glaucodaemon (Dyar \& Shannon) \\
\hline & Trichoprosopon & & Tr. digitatum (Rondani) \\
\hline & Wyeomyia & Phoniomyia & Wy. edwardsi (Lane \& Cerqueira) \\
\hline & & & Wy. splendida (Bonne-Wepster \& Bonne) \\
\hline & & & Wy, palmata / diabolica \\
\hline & & Dodecamyia & Wy, aphobema Dyar \\
\hline & & Dendromyia & Wy. complosa (Dyar) \\
\hline & & & Wy. jocosa (Dyar \& Knab) \\
\hline & & $\cdots$ & Wy. aporonoma Dyar \& Knab \\
\hline & & & Wy, compta Senevet \& Abonnenc \\
\hline & & & Wy, argenteorostris (Bonne-Wepster \& Bonne) \\
\hline & & & Wy, flui (Bonne-Wepster \& Bonne) \\
\hline Uranotaeniini & Uranotaenia & Uranotaenia & Ur. geometrica Theobald \\
\hline
\end{tabular}


Limatus e Uranotaenia); com armadilha Malaise (Culex, Johnbelkinia, Limatus, Sabethes, Trichoprosopon, Uranotaenia, Wyeomyia); e varredura com puçá Anopheles, Coquillettidia, Culex, Haemagogus, Limatus, Ochlerotatus, Psorophora, Sabethes e Wyeomyia). Os imaturos foram coletados em bromélias (Wyeomyia), poças no solo (Aedes, Anopheles e Culex) e em cavidades em pedras (Culex e Ochlerotatus) (Tab. 2).

\section{DISCUSSÃO}

Cerqueira (1961) e Xavier \& Mattos (1976), aparentemente com base nos registros de Deane (1947), assinalaram a presença de 13 espécies coletadas em São Gabriel da Cachoeira (Uaupés) (Tabela 3). Porém, o primeiro registro da presença de An. albitarsis na região foi atribuído por Xavier \& Mattos (1976) à Cerqueira (1961) pois a mesma não foi registrada por Deane (1947). Da mesma forma, Xavier \& Mattos (1976) reconhecem a ocorrência de An. matogrossensis, registrada por Deane (1947), a qual não foi incluida por Cerqueira (1961).

Dentre as espécies coletadas no presente estudo, apenas $A n$. (Stethomyia) nimbus e An. (Nys.) oswaldoi haviam sido assinalados anteriormente (Tabela 3). Em relação ao An. (Nys.) oswaldoi, vale assinalar que, recentemente foram observadas evidencias moleculares que demonstraram que o táxon reconhecido como An. oswaldoi corresponde, provavelmente, a complexo de quatro espécies crípticas (Marrelli et al., 2000b). Até o momento, é possível separar An. oswaldoi de An. konderi mediante o emprego de caracteres anatômicos das estruturas da genitália masculina e, provavelmente, por caracteres moleculares relacionados à sequiência de nucleotídeos do gene ITS2 do DNA do ribossomo (Marrelli et al., 2000a). Acresce considerar que a separação de An. oswaldoi e An. konderi parece não ser possível por caracteres dos ovos observados em microscópio eletrônico de varredura (Lounibos et al., 1997). Em relação à susceptibilidade do An. oswaldoi e An. konderi ao Plasmodium vivax, Marrelli et al. (2000a) demonstraram que em condições de laboratório, o An. oswaldoi parece ser mais suscetível do que o An. konderi, apresentando taxas de infecção mais elevadas e a presença de esporozoítos nas glândulas salivares. Os espécimes coletados na localidade de Querari foram, tentativamente, identificados como pertencentes à espécie $A n$. oswaldoi pela ausência de indivíduos do sexo masculino.

Temos assim 42 novos registros de espécies no município de São Gabriel da Cachoeira. Vale mencionar que os registros anteriores não foram resultados de coletas realizadas com a finalidade do conhecimento da fauna de Culicidae, mas tinham, principalmente, objetivos epidemiológicos (Deane, 1947; Cerqueira, 1961). Dessa maneira, podese sugerir que essa possa ser uma das causas de haver somente espécies do gênero Anopheles nos registros da literatura relativos àquela localidade.

Entre as espécies capturadas, os 
Tabela 2. Relação das espécies de Culicidae, adulto e imaturos, coletadas em Querari, Estado do Amazonas, Brasil, indicando o método de coleta e o tipo de criadouro.

\begin{tabular}{|c|c|c|c|c|}
\hline Espécie & Puçá & CDC & Malaise & Criadouro \\
\hline Ae. arborealis & $x$ & & & \\
\hline Ae. fluviatilis & & & & poças no solo \\
\hline Ae. hastatus & $x$ & & & poças no solo \\
\hline Ae. hastatus / oligopistus & $x$ & & & \\
\hline Ae. hortator & $x$ & & & poças em pedras \\
\hline Ae. serratus & $x$ & & & \\
\hline Ae. serratus / nubilus & $x$ & & & \\
\hline An. nimbus & & & & poças no solo \\
\hline An. oswaldoi & $x$ & $x$ & & \\
\hline Cq. arribalzagai & $x$ & & & \\
\hline Cx. alogistus & & & & poças no solo \\
\hline Cx. anduzei / urichil & $x$ & $x$ & & \\
\hline Cx. coppenamensis (prox.) & & $x$ & & \\
\hline Cx. crybda & $x$ & $x$ & & \\
\hline Cx. declarator & $x$ & & & \\
\hline Cx. distinguendus & & & & poças no solo \\
\hline Cx. educator (prox.) & & $x$ & & \\
\hline Cx. guyanensis / clastrieri & & $x$ & & \\
\hline Cx. lacertosus & $x$ & $x$ & & poças no solo \\
\hline Cx. mollis & $x$ & $x$ & & \\
\hline Cx. pedroi & $x$ & $x$ & & \\
\hline Cx. surinamensis & & & & poças em pedras \\
\hline Cx. theobaldi & & $x$ & $x$ & \\
\hline Cx. urichii & $x$ & & & \\
\hline Cx. vaxus & $x$ & $x$ & $x$ & \\
\hline Cx. vaxus (morfotipo 1) & & $x$ & & \\
\hline
\end{tabular}


Tabela 2. Continuação

\begin{tabular}{|c|c|c|c|c|}
\hline Espécie & Puçá & CDC & Malaise & Criadouro \\
\hline Cx. vaxus (morfotipo 2) & & $x$ & & \\
\hline Cx, vomerifer & $x$ & $x$ & & \\
\hline Cx. zeteki & & $x$ & & \\
\hline Cx. grupo Coronator & $x$ & & $x$ & \\
\hline Cx. evansae (prox.) & $x$ & $x$ & & \\
\hline Cx. (Cux.) spp. & & & & solo; poças em pedras \\
\hline CX. (Mel.) spp. & $x$ & $x$ & & poças no solo \\
\hline Ha. leucocelaenus (prox.) & $x$ & & & \\
\hline Jo. longipes & & & $x$ & \\
\hline Li. durhami & & & $x$ & \\
\hline Li. flavisetosus & $x$ & $x$ & $x$ & \\
\hline Li. pseudomethisticus & $x$ & & $x$ & \\
\hline Ps. albigenu & $x$ & & & \\
\hline Ps. albipes & $x$ & & & \\
\hline Ps. amazonica & $x$ & & & \\
\hline Ps, ferox & $x$ & & & \\
\hline Sa. albiprivus & & & $x$ & \\
\hline Sa. cyaneus & & & $x$ & \\
\hline Sa. glaucodaemon & $x$ & & & \\
\hline Sa. purpureus & & & $x$ & \\
\hline Tr. digitatum & $\mathrm{X}$ & & $x$ & \\
\hline Ur. geometrica & $x$ & $x$ & $x$ & \\
\hline Wy. aphobema & & & & bromélias terrestres \\
\hline Wy. aporonoma & $x$ & & $x$ & \\
\hline Wy, argenteorostris & & & & bromélias epifitas \\
\hline Wy. complosa & $x$ & & $x$ & \\
\hline Wy. compta & & & & bromélias epifitas \\
\hline Wy. edwardsi & & & & bromélias epífitas \\
\hline Wy. jocosa & $x$ & & $x$ & \\
\hline Wy. flui & $x$ & & $x$ & \\
\hline Wy. palmata / diabolica & $x$ & & & \\
\hline Wy. splendida & $x$ & & & \\
\hline
\end{tabular}


Tabela 3. Comparação das espécies registradas em duas localidades do Município de São Gabriel da Cachoeira, Estado do Amazonas, Brasil.

\begin{tabular}{|c|c|c|}
\hline Espécie & São Gabriel / Uaupés & Querari $^{d}$ \\
\hline Ae. arborealis & & $x$ \\
\hline Ae, fluviatilis & & $x$ \\
\hline Ae. hastatus & & $x$ \\
\hline Ae. hastatus / oligopistus & & $x$ \\
\hline Ae. hortator & & $x$ \\
\hline Ae. serratus & & $x$ \\
\hline Ae. serratus / nubilus & & $x$ \\
\hline An. albitarsis I.s. & $X^{b c}$ & \\
\hline An, braziliensis & $X a b c$ & \\
\hline An. darlingi & $X a b c$ & \\
\hline An. eiseni & $X$ abo & \\
\hline An. kompi & $X a b c$ & \\
\hline An. mattogrossensis & $x$ ac & \\
\hline An. mediopunctatus & $X \otimes B C$ & \\
\hline An. nimbus & $X^{a b c}$ & $x$ \\
\hline An. nuneztovari & $X a b c$ & \\
\hline An. oswaldoi & $X a b c$ & $x$ \\
\hline An. peryassui & $X$ abc & \\
\hline An. shannoni & $X \pm b c$ & \\
\hline An. thomasi & $X$ a b $c$ & \\
\hline An. triannulatus davisi & $X a b=$ & \\
\hline Cq. arribazagai & & $x$ \\
\hline Cx. alogistus & & $x$ \\
\hline Cx. anduzeilurichii & & $x$ \\
\hline Cx. coppenamensis (prox.) & & $x$ \\
\hline Cx. grupo coronator & & $x$ \\
\hline Cx. crybda & & $x$ \\
\hline Cx. declarator & & $x$ \\
\hline$C x$, distinguendus & & $x$ \\
\hline Cx. educator (prox.) & & $x$ \\
\hline Cx. evansae (prox.) & & $x$ \\
\hline
\end{tabular}


Tabela 3. Continuação

\begin{tabular}{|c|c|c|}
\hline Espécie & São Gabriel / Uaupés & Querarid \\
\hline Cx. guianensis / clastrieri & & $x$ \\
\hline Cx. lacertosus & & $x$ \\
\hline Cx. mollis & & $x$ \\
\hline Cx, pedroi & & $x$ \\
\hline Cx. surinamensis & & $x$ \\
\hline Cx. theobaldi & & $x$ \\
\hline Cx. urichii & & $x$ \\
\hline Cx, vaxus & & $x$ \\
\hline Cx. vaxus (morfotipos 1 e 2 ) & & $x$ \\
\hline Cx. vomerifer & & $x$ \\
\hline Cx. zeteki & & $x$ \\
\hline Hg. leucocelaenus (prox.) & & $x$ \\
\hline Jh. longipes & & $x$ \\
\hline Li. durhami & & $x$ \\
\hline Li. flavisetosus & & $x$ \\
\hline Li. pseudomethisticus & & $x$ \\
\hline Ps. albigenu & & $x$ \\
\hline Ps. albipes & & $x$ \\
\hline Ps. amazonica & & $x$ \\
\hline Ps. ferox & & $x$ \\
\hline Sa albiprivus & & $x$ \\
\hline Sa. cyaneus & & $x$ \\
\hline Sa. glaucodaemon & & $x$ \\
\hline Sa. purpureus & & $x$ \\
\hline Tr. digitatum & & $x$ \\
\hline Ur. geometrica & & $x$ \\
\hline Wy. aphobema & & $x$ \\
\hline Wy. aporonoma & & $x$ \\
\hline Wy. argenterostris & & $x$ \\
\hline Wy. complosa & & $x$ \\
\hline Wy. compta & & $x$ \\
\hline Wy. edwardsi & & $x$ \\
\hline Wy, flui & & $x$ \\
\hline Wy, jocosa & & $x$ \\
\hline Wy palmata / diabolica & & $x$ \\
\hline Wy. splendida & & $x$ \\
\hline
\end{tabular}

- Deane (1947)

Cerqueira (1961)

- Xavier \& Mattos (1976)

Este trabalho 
membros do subgênero Melanoconion de Culex tiveram maior representação. Sabe-se que o subgênero Melanoconion reúne representantes dos quais têm-se levantado hipóteses sobre a responsabilidade vetora de agentes infeciosos (Natal, 1981). Estes agentes infecciosos no estado atual dos conhecimentos, são representados essencialmente por arbovírus. Algumas espécies de Melanoconion foram encontradas naturalmente infectadas com múltiplos vírus, apresentando portanto competência para atuarem como vetores biológicos desses agentes infecciosos (Srihongse et al., 1967). A presença de $C x$. (Mel.) pedroi e de $C x$. (Mel.) vomerifer, espécies identificadas como vetoras de arbovírus na região amazônica (Srihongse et al., 1967; Vasconcelos et al., 1991), pode ter implicações epidemiológicas para a região. Representantes de Culex vomerifer foram encontrados naturalmente infectados com vírus da Família Bunyaviridae (Guama, Moju, Ananindeua, Caraparu, Ossa, Vinces. Madrid, Murutucu e Itaqui) (Shope et al., 1988). Portanto, é possível sugerir que esteja ocorrendo a circulação desses agentes infeciosos na região com possibilidade de envolvimento ocasional do homem.

Outro grupo que merece destaque, com maior representação nas coletas do que os demais grupos de Culicidae, são os mosquitos silvícolas da tribo Sabethini, em especial o gênero Wyeomyia. Resultados semelhantes foram observados em ambientes com características similares localizado na Serra do Mar, estado de São Paulo (Guimarães et al., 2000): regiões de mata primária com temperatura e umidade relativa elevadas e cobertura vegetal densa, rica em Bromeliacea, contendo estes ambientes muitos locais tais como, internódios de bambu, buracos em árvores e axilas de folhas de diferentes espécies, que servem de local de oviposição e desenvolvimento dos estádios imaturos de Sabethini.

É importante considerar que um maior conhecimento da fauna de $\mathrm{Cu}$ licidae da região amazônica permitirá a obtenção de informações básicas sobre a diversidade faunística, distribuição e a variedade de ecossistemas onde os mosquitos ocorrem, fornecendo assim conhecimentos para estudos de controle das doenças que afetam os homens e animais cujos agentes infeciosos são veiculados por mosquitos. É provável que outros pesquisadores, especialmente os ligados a órgãos de saúde, tenham registros de distribuição de Culicídeos no município de São Gabriel da Cachoeira, que não estão disponíveis por não terem sido publicados. Esperamos que este trabalho sirva como estímulo para que as informações existentes sejam divulgadas aumentando assim nosso conhecimento da fauna amazônica.

\section{AGRADECIMENTOS}

Nossos estudos não teriam sido possíveis sem o apoio do Comando Militar da Amazônia, principalmente do $2^{\circ}$ Pelotão Especial de Fronteira 
(Querari), do Sr. Roberto Stieger Leite (INPA), e do Tenente-Coronel-Aviador Otelo Guimarães (Força Aérea Brasileira). Agradecemos ao Sr. João Vidal (INPA) pelo colaboração nas coletas do material e, em especial, ao Prof. Dr. Oswaldo P. Forattini por permitir o uso dos recursos logísticos do NUPTEM, pela ajuda nas identificações e sugestões para a melhoria do manuscrito.

\section{Bibliografia citada}

Berlin, O.G.W.; Belkin, J.N. 1980. Mosquito Studies (Diptera, Culicidae) XXXVI. Subgenera Aedinus, Tinolestes and Anoedioporpa of Culex. Contributions of The American Entomological Institute (Ann Arbor), 17(2): 1-104.

Cerqueira, N.L. 1961, Distribuição geográfica dos mosquitos da Amazônia (Diptera, Culicidae, Culicinae), Revista Brasileira de Entomologia, 10(111-168.

Deane, L.M. 1947. Observações sôbre a malária na Amazônia brasileira. Revista do Serviço Especial de Saúde Pública M.E.S., 1(1): 3-59.

Forattini, O.P. 1965. Entomologia Médica Vol.2 Culicini: Culex, Aedes e Psorophora. Universidade de São Paulo, São Paulo. 506 p.

Frank, J.H.; Lounibos, L.P. (Eds) 1983. Phytotelmata. Terrestrial plants as hosts for aquatic insect communities. Plexus Publishing, Medford, New Jersey.

Guimarães, A.E.; Mello, R.P.; Lopes, C.M.; Gentile, C. 2000. Ecology of mosquitoes (Diptera: Culicidae) in Areas of Serra do Mar State Park, State of São Paulo, Brazil. I- Monthly frequency and climatic factors. Memorias do Instituto Oswaldo Cruz, 95(1): 1-16.

Koopen, W. 1948. Climatologia; com un estudio de los climas de la tierra. Fondo de Cultura Economica, Mexico. 478 p.

Lane, J. 1953. Neotropical Culicidae. Vol. 2
Tribe Culicini, Deinocerites, Uranotaenia, Mansonia, Orthopodomyia, Aedomyia, Aedes, Psorophora, Haemagogus, tribe Sabethini, Trichoprosopon, Wyeomyia, Phoniomyia, Limatus and Sabethes. University of São Paulo, São Paulo. 554-1112 p.

Lounibos, L.P.; Duzak, D.; Lynley, J.R. 1997. Comparative egg morphology of six species of the Albimanus section of Anopheles (Nyssorhynchus) (Diptera: Culicidae). Journal Of Medical Entomology, 34(2): 136-155.

Marrelli, M.T.; Honorio, N.A.; Flores, C.M.; Lourenço-de-Oliveira, R.; Marinotti, O.; Kloctzel, J.K. 2000a. Comparative susceptibility of two members of the Anopheles oswaldoi complex, An. oswaldoi and An. konderi, to infection by Plasmodium vivax. Transactions of The Royal Society Of Tropical Medicine and Hygiene, 93: 381-384.

Marrelli, M.T.; Malafronte, R.S.; Flores, C.M.; Lourenço-de-Oliveira, R.; Kloctzel, J.K.; Marinotti, O. 2000b. Sequence analysis of the second internal transcribed spacer of ribosomal DNA in Anopheles oswaldoi (Diptera: Culicidae), Journal Of Medical Entomology, 36: 679-684.

Natal, D. 1981. Importância epidemiológica do subgênero Melanoconion (Diptera: Culicidae). Dissertação de Mestrado, Faculdade de Saúde Pública da USP, São Paulo.

Nielsen, L.T. 1980. The current status of mosquito systematics. Mosquito Systematics, 12(1): 1-6.

RADAMBRASIL 1976. Folha NA.I9 Pico da Neblina: geologia, geomorfologia. pedologia, vegetação e uso potencial da terra. DNPM - Ministério das Minas e Energia, Rio de Janeiro. 380 p.

Shope, R.E.; Woodhall, J.P.; Da Rosa, A.T. 1988. The epidemiology of diseases caused by viruses in Groups $\mathrm{C}$ and Guama (Bunyaviridae), In: Monath, T. P. (Ed) The Arboviruses: epidemiology and ecology. CRC Press, Boca Raton, FL, p. 37-52.

Srihongse, S.; Scherer, W.F.; Galindo, P. 1967. Detection of arboviruses by sentinel ham- 
sters during the low period of transmission. American Journal of Tropical Medicine And Hygiene, 16(4): 519-530.

Vasconcelos, P.F.C,; Travassos da Rosa, J.F.S.; Degallier, N.; Pinheiro, F.d.P.; Sá Filho, G.C. 1991. Epidemiologia das encefalites por arbovírus na Amazônia Brasileira. Revista do Instituto de Medicina Tropical de São Paulo, 33(465-476.

Ward, R.A. 1992. Third Supplement to "A Catalog of the Mosquitoes of the World" (Diptera: Culicidae). Mosquito Systematics, 24(3): 177-230.

Xavier, S.H.; Mattos, S.S. 1976. Geographical distribution of Culicinae in Brazil - IV. State of Amazonas (Diptera, Culicidae). Mosquito Systematics, 8(4): 386-412.

Zavortink, T.J. 1979. Mosquito Studies (Diptera: Culicidae) XXXV. The new Sabethine genus Johnbelkinia and a preliminary reclassification of the composite genus Trichoprosopon. Contributions of the American Entomological Institute, 17(1): 1-61. 